\title{
Sportfachzeitschriften aus dem Hofmann-Verlag
}

\section{Internationale Zeitschrift für Sportpädagogik International Journal of Physical Education Official Magazine of ICHPER}

Hauptschriftlelter: Prof. Dr. Herbert Haag

In dieser Zeitschrift wird versucht, neben anderen Theoriefeldern, hauptsächlich sportpädagogische Fragestellungen (,physical education“) zu berücksichtigen, nicht zuletzt auf Grund der engen Verbindung zu ICHPER. Diese Ausrichtung auf Sportpădagogik und fur dieses Theoriefeld relevante Fragestellungen sollen stärker betont werden, so daB die Sportpădagogik aúf internationaler Ebene hiermit ein eigenes wissenschaftliches Diskussionsforum erhălt. Die Beitrăge erscheinen in deutscher oder englischer Sprache. Die Hauptbeitrăge erhalten zusätzlich spanische und französische Zusammenfassungen.

Zweimal jährlich mit 16 Seiten „Lehrhilfen fü die Leibeserziehung in Entwicklungsländern" in englischer und spanischer Sprache.

\section{Motorik - Zeitschrift für Motopädagogik und Mototherapie}

Offizielles Organ des Aktionskreises Psychomotorik e.V.

Geschäftsführender Redakteur: Hans-Jürgen Müller

Verantwortlicher Redakteur: Prof. Dr. Friedhelm Schilling

Redaktion: Prof. Dr. Eberhard Hildenbrandt, Heinz Mechling, Prof. Dr. Gerhard Neuhåuser, Prof. Dr. Heinrich Oepen, Prof. Dr. Volker Rittner

Diese vierteljăhrlich erscheinende Zeltschrift vermittelt Sportpådagogen, Bewegungstherapeuten, Lehrern, Erziehern, Heilpådagogen, Psychologen, Kinderärzten, Jugendpsychiatern und Krankengymnasten mit wissenschaftlichen und praktischen Beiträgen uber Neuromotorik, Sensomotorik, Psychomotorik und Soziomotorik wertvolle Grundlagen, Anregungen und Empfehlungen.

\section{sportunterricht Monatsschrift zur Wissenschaft und Praxis des Sports}

Offizielles Organ des Deutschen Sportlehrerverbandes (DSLV) mit Nachrichten der Deutschen Vereinigung für Sportwissenschaft (DVS).

Schriftleiter: Prof. Dr. Gerhard Hecker.

Redaktionskollegium: Prof. Dr. Helmut Digel, Prof. Dr. Ulrich Göhner, Dr. Ulrich Joeres, Prof. Doris Kupper, Prof. Dr. Renate Scholtzmethner, Prof. Dr. Roland Singer, Prof. Dr. Willibald Weichert.

Die Zeitschrift sportunterricht will wissenschaftliche Untersuchungen und Betrachtungen an der Berufspraxis des Sportlehrers orientieren, um dem Leser nicht nur abstrakte Einsichten, sondern vielmehr Entscheidungshilfen und Handlungskriterien zu vermitteln.

Ständige Beilage dieser Zeitschrift sind die Lehrhilfen für den sportunterricht, die auch gesondert bezogen werden können.

\section{S P ORT W I S S E N S C HAF T 11. Jahrgang 1981/1}

Herausgegeben vom Deutschen Sportbund, vom AusschuB Deutscher Leibeserzieher und vom Bundesinstitut für Sportwissenschaft.

(c) 1981 by Verlag Karl Hofmann, 7060 Schorndorf. Alle Rechte, auch das der Übersetzung in fremde Sprachen, vorbehalten. Nachdruck und Vervielfältigungen jeder Art nur mit Genehmigung des Verlags.

Beilagenhinweis: Einer Teilauflage dieser Ausgabe liegt ein Prospekt „Sportunterricht im Sommerhalbjahr" des Hofmann-Verlags bei. 